\title{
ON FIXED POINTS OF LINEAR CONTRACTIONS
}

\author{
HEYDAR RADJAVI AND PETER ROSENTHAL
}

\begin{abstract}
It is shown that a weakly closed convex semigroup of linear contractions on a separable Hilbert space has a common fixed point other than 0 if the operator 0 is not in the semigroup.
\end{abstract}

We prove a theorem on existence of common fixed points for certain convex semigroups of linear operators on Banach spaces. The special case where the semigroup is a group follows easily from Kakutani's well-known theorem [4, 5, 6] and also, as discussions with P. Milman revealed, from the work of Brodskii and Milman [1]. Similarly, in the case where the semigroup is commutative, our result is a corollary of a special case of the Markov-Kakutani theorem [3, 4]. Nonetheless, it appears that the results and corollaries given below have not been noticed before. Corollary 4, for example, gives a sufficient condition that $\bigvee_{n=N}^{\infty}\left\{A^{n}\right\}$ be the same for all $N$.

The applications of the fixed-point theorem that we consider concern operators on Hilbert space, but it seems worthwhile to state the theorem more generally.

THEOREM 1. Let $\mathscr{X}$ be a strictly convex reflexive Banach space, and let $\mathscr{S}$ be a weak operator closed separable convex semigroup of linear contractions on $\mathscr{X}$. Then the operators in $\mathscr{S}$ have a common fixed point other than 0 if and only if the operator 0 is not in $\mathscr{S}$.

Proof. Clearly, if the operator 0 is in $\mathscr{S}$, then the only common fixed point is 0 .

To prove the converse first recall that $\left(T_{\alpha}\right) \rightarrow T$ in the weak operator topology if and only if $\phi\left(T_{\alpha} x\right) \rightarrow \phi(T x)$, for each $\phi \in \mathscr{X}^{*}$ and $x \in \mathscr{X}$. We require the fact that the unit ball of $\mathscr{B}(\mathscr{X})$ is weak operator compact; this can be proven as in the better-known case of Hilbert space. (That is, consider the Cartesian product of the closed balls of radius $\|x\|$ in $\mathscr{X}$, indexed by $\mathscr{X}$, where each ball is given the weak topology).

Let $\left\{T_{n}\right\}_{n=1}^{\infty}$ be a countable weak operator dense subset of $\mathscr{S}$; it obviously suffices to find a common fixed point for the $\left\{T_{n}\right\}$. Let

$$
T=\sum_{n=1}^{\infty} \frac{1}{2^{n}} T_{n}
$$

Received by the editors December 27, 1983 and, in revised form, July 27, 1984.

1980 Mathematics Subject Classification. Primary 47D05, 27D20; Secondary 54H25, 47H10. 
this series converges in the norm topology (hence also in the weak operator topology) of $\mathscr{B}(\mathscr{X})$, and the closed convexity of $\mathscr{S}$ implies $T \in \mathscr{S}$. Now $T$ defines a mapping of $\mathscr{S}$ into itself by $T(S)=T S$ for $S \in \mathscr{S}$ ( $\mathscr{S}$ is a semigroup). Since $\mathscr{S}$ is a compact convex set, Schauder's fixed point theorem yields an operator $S_{0} \in \mathscr{S}$ such that $T S_{0}=S_{0}$. Choose $x \in \mathscr{X}$ such that $S_{0} x \neq 0$. Then

$$
\sum_{n=1}^{\infty} \frac{1}{2^{n}} T_{n} S_{0} x=S_{0} x \text {. }
$$

For each $n_{0}$,

$$
\begin{gathered}
\left\|\sum_{n \neq n_{0}} \frac{1}{2^{n}} T_{n} S_{0} x+\frac{1}{2^{n_{0}}} T_{n_{0}} S_{0} x\right\|=\left\|S_{0} x\right\|, \\
\left\|\sum_{n \neq n_{0}} \frac{1}{2^{n}} T_{n} S_{0} x\right\| \leqslant\left(\sum_{n \neq n_{0}} \frac{1}{2^{n}}\right)\left\|S_{0} x\right\|,
\end{gathered}
$$

and

$$
\left\|\frac{1}{2^{n_{0}}} T_{n_{0}} S_{0} x\right\| \leqslant \frac{1}{2^{n_{0}}}\left\|S_{0} x\right\|
$$

imply that the above inequalities are equations, so the strict convexity of $\mathscr{X}$ implies that $T_{n_{0}} S_{0} x$ is a multiple of $\sum_{n \neq n_{0}} T_{n} S_{0} x / 2^{n}$. Hence, $T_{n_{0}} S_{0} x$ is a multiple of $S_{0} x$. (Recall that $\mathscr{X}$ strictly convex means that $\left\|x_{1}+x_{2}\right\|=\left\|x_{1}\right\|+\left\|x_{2}\right\|$ implies $\left\{x_{1}, x_{2}\right\}$ is linearly dependent). Thus, $T_{n} S_{0} x$ is a multiple of $S_{0} x$ for every $n$. But $\left\{\lambda_{n}\right\}$, complex numbers, satisfying $\sum_{n=1}^{\infty} \lambda_{n} / 2^{n}=1$ and $\left|\lambda_{n}\right| \leqslant 1$ for all $n$ implies $\lambda_{n}=1$ for all $n$, so $T_{n} S_{0} x=S_{0} x$ for all $n$. Therefore, $S_{0} x$ is a common fixed point for $\left\{T_{n}\right\}$ and, hence, for $\mathscr{S}$.

REMARK. As the referee has kindly pointed out, the above proof is similar to a proof given by R. E. Bruck, Jr., Properties of fixed-point sets of nonexpansive mappings in Banach spaces, Trans. Amer. Math. Soc. 179 (1973), 251-262.

Corollary 1. A weakly closed convex semigroup of contractions on a separable Hilbert space has a common fixed point other than 0 if and only if it does not contain the operator 0 .

Proof. A Hilbert space satisfies all the hypotheses on $\mathscr{X}$ in Theorem 1. Also, the unit ball of operators on a separable Hilbert space is a separable metrizable space in the weak operator topology, so every semigroup of contractions is separable.

For the next two corollaries let $\mathscr{S}$ be a weakly closed convex semigroup of contractions on a separable Hilbert space.

Corollary 2. Let $\mathscr{M}$ denote the set of common fixed points of members of $\mathscr{S}$; then $\mathscr{S}$ contains the orthogonal projection onto $\mathscr{M}$.

Proof. As is well known, $\|T\| \leqslant 1$ and $T x=x$ implies $T^{*} x=x$ (begin an orthonormal basis with $x /\|x\|$ and represent $T$ with respect to it). Thus, $\mathscr{M}$ reduces every operator in $\mathscr{S}$. Now $\mathscr{S} \mid \mathscr{M}^{\perp}$ is a weakly closed convex semigroup of contractions on $\mathscr{M}^{\perp}$. Since the only common fixed point of $\mathscr{S} \mid \mathscr{M}^{\perp}$ is $\{0\}$, Corollary 1 
implies that the 0 operator is in $\mathscr{S} \mid \mathscr{M}^{\perp}$. Let $P \in \mathscr{S}$ be such that $P \mid \mathscr{M}^{\perp}=0$; since $P \mid \mathscr{M}$ is the identity, $P$ is the projection on $\mathscr{M}$.

COROLlaRY 3. If $\mathscr{S}$ is not the semigroup consisting only of the identity, then some operator in $\mathscr{S}$ has nontrivial nullspace.

Proof. By Corollary 2, if no operator in $\mathscr{S}$ has nullspace, then the set of common fixed points is the entire space.

The next result is a corollary of Theorem 1 in some cases but not in all. The proof, however, is contained in that of Theorem 1.

THEOREM 2. If $\mathscr{S}$ is a weak operator closed bounded convex set of linear operators on a reflexive space and $0 \notin \mathscr{S}$, then 1 is an eigenvalue of every operator $T$ with the property that $S \in \mathscr{S}$ implies $T S \in \mathscr{S}$.

Proof. Let $T$ be as stated. By Schauder's theorem, $T S_{0}=S_{0}$ for some $S_{0} \in \mathscr{S}$. Choose $x$ such that $S_{0} x \neq 0$; then $T S_{0} x=S_{0} x$, so 1 is an eigenvalue of $T$.

COROLlARY 4. If $A$ is an injective operator on Hilbert space, and if there is a $k$ such that $\left\|(1+A)^{n}\right\| \leqslant k$ for every positive integer $n$, then the weakly closed linear span of $\left\{A^{n}: n \geqslant N\right\}$ is the same for all nonnegative integers $N$.

Proof. Let $T=1+A$ and let $\mathscr{S}$ be the weakly closed convex hull of $\left\{T^{n}: n \geqslant 1\right\}$. Since $A$ has no nullspace, $T$ has no fixed points other than 0 . By Theorem 2, $0 \in \mathscr{S}$. Thus, given any weak operator neighborhood $\mathscr{W}$ of 0 there is a collection of nonnegative numbers $\left\{\lambda_{j}\right\}_{j=1}^{m}$ such that $\sum_{j=1}^{m} \lambda_{j}=1$ and $\sum_{j=1}^{m} \lambda_{j} T^{j} \in \mathscr{W}$. Then $\sum_{j=1}^{m} \lambda_{j} T^{j}$ has the form $1+\sum_{j=1}^{m} \lambda_{j} p_{j}(A)$ for suitable polynomials $p_{j}$ without constant terms. It follows that 1 is in the weak closure of the linear span of $\left\{A^{n}: n \geqslant 1\right\}$. Thus, $A$ is also in the weak closure of the linear span of $\left\{A^{n}: n \geqslant 2\right\}$ (multiplication is separately weakly continuous in each variable), and the corollary follows by a trivial induction.

\section{REFERENCES}

1. M. S. Brodskii and D. P. Milman, On the centre of a convex set, Dokl. Akad. Nauk SSSR (N.S.) 59 (1948), 837-840. (Russian)

2. M. M. Day, Normed linear spaces, Springer-Verlag, Berlin, 1962.

3. J. Dugundji and A. Granas, Fixed point theory, Vol. I, PWN, Warsaw, 1982.

4. N. Dunford and J. T. Schwartz, Linear operators, Part I, Interscience, New York, 1957.

5. S. Kakutani, Two fixed-point theorems concerning bicompact convex sets, Proc. Imp. Acad. Tokyo 14 (1938), 242-245.

6. D. R. Smart, Fixed point theorems, Cambridge Univ. Press, London, 1974.

Department of Mathematics, Dalhousie University, Halifax B3H 4H8, Nova Scotia, Canada

Department of Mathematics, University of Toronto, Toronto M5S 1A7, Ontario, Canada 